\title{
II Green Pass all'Accademia della Crusca (e altrove), ovvero per una storia del Green Pass
}

\author{
Salvatore Claudio Sgroi
}

PUBBLICATO: 25 SETTEMBRE 2021

\section{L'evento cruscante}

Chi si collega col sito della Crusca ha modo di leggere il seguente avviso per quanti vogliano recarsi nella sede dell'Accademia:

\begin{abstract}
Avvisiamo che dal 6 agosto 202I, come stabilito dal Decreto Legge del 23 luglio 202I n. I05, articolo 3 comma 3 lettera c, l'accesso alla biblioteca e all'archivio, e la partecipazione a convegni, seminari, visite guidate e qualsiasi altra manifestazione, è consentito esclusivamente a soggetti muniti di certificazione verde Covid-Ig (Green Pass), fatto salve le esclusioni e le esenzioni previste dallo stesso Decreto.

Il personale dell'Accademia è incaricato, all'ingresso, di accertare il possesso dei requisiti richiesti.
\end{abstract}

\section{I.I. La strategia bilingue}

Una soluzione equilibrata, quella della Crusca, dinanzi al problema dello stranierismo - Green Pass con l'adozione della strategia bilingue, che da un lato riconosce l'esistenza del diffusissimo anglicismo, che viene però fatto precedere da un traducente / equivalente italiano - certificazione verde Covid-19-, inequivoco per la presenza anche dell'apposizione "Covid-19".

\section{I.I.I. Il Green Pass ufficiale}

La strategia della Crusca è quella del Green Pass digital-cartaceo rilasciato ufficialmente agli italiani, che prevede la dizione bilingue nella variante:

$$
\text { "Certificazione verde COVID-19" }
$$

con sotto quella inglese:

$$
\text { "EU Digital COVID Certificate", }
$$

dove però non appare la dizione green pass.

Come dire una soluzione diglottica: oralmente (varietà Bassa) "Green Pass" vs scritto (varietà Alta): "Certificazione verde COVID-I9".

\section{I.2. Il presidente della Crusca e il Green Pass}

Claudio Marazzini, in quanto presidente della Crusca, è stato intervistato da Paolo Martini di Adn Kronos, il 23 luglio 202I, sul Green Pass, il cui testo riprendeva nel titolo le parole di Marazzini: 
Accademia della Crusca: No "green pass", meglio dire "certificato Covid".

Il presidente Claudio Marazzini: «Bene anche "certificato vaccinale" o "passaporto vaccinale"».

E così continuava, con l'indicazione prescrittiva e neopuristica nell'ottica del gruppo Incipit:

Evitare di usare "green pass" e preferire "certificato Covid", seguendo il nome ufficiale dell'Unione Europea riportato nello stesso attestato, o i possibili sostituti più generici "certificato vaccinale" o "passaporto vaccinale".

La prescrizione è giustificata data la polisemia equivoca dell'anglicismo:

Il termine "green pass", [...], mutuato dall'esperienza degli Usa, appare "infelice, equivoco e polisemico: si fa ancora una volta ricorso alla lingua inglese, ma si usa un anglismo che non è affatto chiaro. Eviterei sempre giochi di prestigio con le parole, perché la polisemia rende poco trasparente ciò di cui si parla".

Più in generale Marazzini "raccomanda 'ancora una volta, nel linguaggio pubblico, termini limpidi' ". E ricorda che "a livello europeo si parla, appunto, di "digital Covid certificate", che può essere facilmente tradotto in italiano con "certificato digitale Covid". Il che costituisce un quarto possibile traducente.

\section{I.2.I. Huffingtonpost.it e "Il Resto del Carlino"}

L'intervista di ADN Kronos è ripresa da altre testate on line. Lo stesso giorno Huffingtonpost.it pubblica un pezzo anonimo intitolato:

Accademia della Crusca "si ribella" al green pass: "Chiamarlo certificato Covid"

Contrarietà a un anglismo "infelice, equivoco e polisemico",

senza dimenticare nel corpo dell'articolo gli altri due traducenti proposti da Marazzini: "certificato vaccinale" e "passaporto vaccinale".

Ne "Il Resto del Carlino" del 25 luglio appare invece un intervento di Piefrancesco Giannangeli intitolato "Certificato Covid. Diciamolo in italiano", nello spirito di "Incipit", che riprende i vari punti affrontati da Marazzini, con i tre succitati traducenti: passaporto vaccinale, certificato vaccinale e certificato Covid. Quest'ultimo è giudicato, si direbbe, dal giornalista "l'espressione che pare essere in questo momento più gettonata", confermata da Google (cfr. più avanti § 6), mentre il "vaccinale sulle altre due formulazioni suona - neopuristicamente per il giornalista - maluccio".

\section{I.2.2. "Il Foglio" e la polemica delirante contro il "Green Pass"}

Su "Il Foglio" del 28 luglio (p. 2) è apparso a nome di Saverio Raimondo un articolo in pura polemica politica con chi è contrario all'adozione del Green Pass, giudicato una "Dittatura sanitaria" (così nel titolo) "dai paladini della libertà". L'autore estende quindi la polemica contro chi è contrario non alla scelta politica ma all'uso dell'anglicismo, come nel caso dell'Accademia della Crusca. E confondendo allegramente - le due prospettive propone un "trattamento sanitario obbligatorio" nei due casi.

\section{Green Pass nell'uso giornalistico}


Se si vuole verificare l'uso reale nella stampa nazionale, per es. ne "la Repubblica" (II settembre) si constata che il sintagma Green Pass ricorre in otto testi non meno di 2I volte:

(i) "Brunetta: Green Pass per gli statali e nelle aziende private". (Alberto Flores d'Arcais, p. I)

(ii) Il ministro: "Green Pass per tutti i settori". (p. 2)

(iii) Renato Brunetta, dice di essere favorevole e intanto indica la via di un Green Pass per tutti i dipendenti.

(iv) E il ministro della Salute Roberto [...] "Lavoreremo per un ulteriore allargamento del Green Pass e valuteremo l'estensione dell'obbligo vaccinale [...]". (ibid.)

(v) Matteo Salvini, segretario della Lega, si dice invece contrario all'estensione del Green Pass a tutti i lavoratori. (ibid.)

(vi) Nelle prossime settimane sarà allargata progressivamente la fascia di cittadini che dovranno munirsi di Green Pass. (Emanuele Lauria, p. 3)

(vii) ha partorito un documento che, appunto, apre all'estensione generalizzata del Green Pass e subito dopo alla vaccinazione obbligatoria. (ibid.)

(viii) Lega e Fdi, contrari all'obbligo vaccinale e scettici sull'allargamento del Green Pass. (ibid.)

(ix) una comune azione in aula di Lega e Fratelli d'Italia, sconfitta dai numeri, per abolire il Green Pass nei ristoranti e per gli under $\mathrm{i} 8$. (ibid.)

(x) Il partito della Meloni è nettamente contrario al Green Pass. (ibid.)

(xi) L'estensione del Green Pass è solo l'ultimo dei punti che ormai dividono il partito di Berlusconi dagli alleati del centrodestra. (ibid.)

(xii) «È il metodo più sicuro - è l'opinione del leghista — più del Green Pass» (Gabriele Bortoloni, ibid.)

(xiii) «Non ci saranno sigle o movimenti, c’è il popolo contro il Green Pass e contro questa dittatura». (Alessandra Ziniti, p. 4)

(xiv) Ma sul Green Pass nella scuola, [...] è ancora un tiro incrociato. (Viola Giannoli, p. 7)

(xv) per lo Snals l'estensione del Green pass comporta "nuovi impegni sulle spalle dei presidi, già gravati dada mancanza di aule e di personale". (ibid.)

(xvi) La buona notizia: a oggi piu del 93\% del personale ha ricevuto almeno una dose e quasi sempre anche un Green Pass. (ibid.)

(xvii) Dopo diciotto mesi proibitivi per i matrimoni, si è scatenata la corsa al "grande si", sia pure con mascherina e invitati muniti di green pass. (Atelier Emé, p. 23)

(xviii) Lennon, Imagine e gli islamisti Green Pass e "Maestri cercando". (Francesco Merlo, p. 27)

(xix) I due filosofi pongono domande etico politiche sul Green Pass. (ibid.)

(xx) Il Green Pass invece non è materia per maestri. (ibid.)

(xxi) [...] impongono le loro varianti del Green Pass. (Federico Rampini, p. 29)

\section{I. Green Pass in "Google libri ricerca avanzata"}

In "Google libri ricerca avanzata" si ritrovano per es. varie attestazioni di Green Pass databili in genere a metà 202I, tra cui:

(i) Emiliano Bezzi, Pasquale Liberatore 202r: $\underline{11}$ green pass in assenza di un obbligo vaccinale. (in Digital green pass. Questioni giuridiche sottese, Key Editore, p. 63)

(ii) AA.VV. 202r: Al via il Green pass Ue. Entra in vigore il Green pass che permette ai titolari, vaccinati, guariti dall'infezione o con un test recente negativo, di viaggiare in tutti i paesi dell'Unione senza doversi sottoporre a tamponi e quarantene. (VACANZE - L'Italia riapre al turismo. Le mete regione per regione, "Il Sole 24 Ore", p. 9)

(iii) OECD 202I: Il settore dei servizi si è ripreso rapidamente in risposta al programma di vaccinazione lanciato dal Governo e alla diffusione del cosiddetto "green pass". (Studi economici dell'OCSE: Italia 2021, p. 26)

(iv) Gilberto Corbellini, Alberto Mingardi 202r: Forse non hanno apprezzato fino in fondo il ritorno alla normalità, se hanno pensato di vincolare nuovamente l'accesso ai locali e agli eventi pubblici 
attraverso il "Green pass". (La societá chiusa in casa, Marsilio)

(v) AA.VV. 202r: si-no-forse, cacciari-agamben, green pass. Registrati comunque in lockdown i I2 brani del disco sono intitolati per lo più a città lontane. (Linus. Settembre 202I, p. 98)

(vi) Ida Dominijanni 2021: sovrano dittatoriale che gli impone il marchio del green pass come Hitler imponeva agli ebrei la stella di David. (2001. Un Archivio: L'11 settembre, la war on terror, la caccia ai virus, Manifestolibri)

(vii) Stefano Nasetti 202r: Medesimo discorso può essere ripetuto per il "green pass" (o passaporto vaccinale) di cui ho già detto. (Fact Checking. La realtà dei fatti, la forza delle idee, ed. Stefano Nasetti p. 298)

\subsection{No Green Pass nell'uso giornalistico}

Due invece i composti animati No Green Pass 'chi è contrario al green pass' nel citato "la Repubblica":

(i) No Vax e_No Green Pass avevano minacciato di bloccare il traffico ferroviario in tutta Italia". (Alessandra Ziniti, p. 4)

(ii) Guido Cappelli, papà di un bimbo delle elementari e firmatario degli appelli No Green Pass, escluso dalla scuola dove doveva firmare alcuni moduli. (Viola Giannoli, p. 7)

\subsection{Anti-Green pass}

Ne "il Fatto quotidiano" (I2 settembre, p. I7) non manca neppure un composto esocentrico come antiGreen pass 'chi è contrario al green pass':

Nelle ultime settimane, alle proteste anti-Green pass si è registrata una partecipazione sempre minore, dopo.

\subsection{L'abbreviazione pass}

Il Green pass alterna anche con l'abbreviazione Pass nel citato art. di Viola Giannoli su "la Repubblica", in almeno 5 ess.:

(i) "Ressa e scelte tardive" L'ultima lite a scuola è sul Pass per i genitori. (titolo, p. 7)

(ii) A controllare i pass a mamme, papà, nonni e tate ci sarà il personale Covid delle scuole. (ibid.)

(iii) La nuova piattaforma per la verifica del Pass del personale è pronta, collaudata dal 4 , debutterà proprio lunedi. (ibid.)

(iv) E se un ragazzo si sente male e un genitore non ha il pass che deve fare? (ibid.)

(v) A Viareggio come a Roma o a Genova il pass negli asili veniva già chiesto, con qualche problema per gli esclusi. (ibid.)

\subsection{Il No Pass}

E non manca il composto animato No Pass "chi è contrario al green pass":

l'indagine milanese che ha sventato il progetto di azioni violente da parte di alcuni esponenti della eterogenea galassia dei No Vax e No Pass. (Alessandra Ziniti, p. 4) 


\section{Il traducente certificazione verde}

Accanto all'uso preponderante di Green Pass (2I ess.) ne "la Repubblica" va rilevata nella stessa sede del quotidiano la presenza con due occorrenze del traducente certificazione verde, ufficiale e avallato dalla Crusca (§§ I.I; I.I.I; I.2; I.2.I):

(i) C'è da attendersi, dunque, che anche oggi in piazza, tra chi protesta contro l'estensione dell'obbligatorietà della certificazione verde, possano esserci frange estremiste. (Alessandra Ziniti p. 4) (ii) L'ultimo scontro riguarda la norma contenuta nel decreto approvato giovedi sera dal Consiglio dei ministri, ovvero l'obbligo di mostrare la Certificazione verde per chiunque entri negli istituti scolastici, dall'asilo in su. (Viola Gianoli p. 7)

E una volta la certificazione verde è abbreviata in Certificazione col richiamo al "semaforo verde o rosso":

(iii) La nuova piattaforma per la verifica del Pass del personale è pronta, collaudata dal 4, debutterà proprio lunedi: il preside o un suo delegato si collegherà al sito del ministero con credenziali riservate e sul profilo della scuola potrà immediatamente verificare ogni giorno lo stato di validità della Certificazione di tutto il personale con un semaforo verde o rosso. In questo secondo caso scatteranno le verifiche. (ibid.)

\section{I. Il traducente certificato verde}

Da rilevare ancora la presenza nello stesso quotidiano del traducente certificato verde con due occorrenze:

(i) Matteo Salvini, segretario della Lega, si dice invece contrario all'estensione del Green Pass a tutti i lavoratori ma deve subire il pressing dei governatori del suo partito, Zaia, Fontana e Fedriga, che continuano a promuovere il certificato verde e a benedirne un ampio utilizzo. (Flores d'Arcais, p. 2)

(ii) L'obiettivo è quello di raggiungere, attraverso lo strumento del certificato verde, una soglia di "protetti" pari all'85-9o per cento degli immunizzati con due dosi. (Emanuele Lauria, p. 3)

Una volta il sintagma è abbreviato, dato il contesto, in certificato:

Il ministro: "Green Pass per tutti i settori". Anche i governatori leghisti per l'estensione del certificato. (A. Flores d'Arcais, catenaccio, p. 2)

\section{I.I. Certificato vaccinale (e anglo-amer. vaccine certificate)}

Un ulteriore traducente nello stesso quotidiano è costituito dal sintagma certificato vaccinale, già

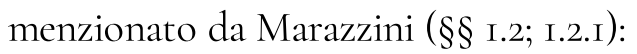

Ora non c'è solo il no all'estensione del certificato vaccinale nell'orizzonte del leader della Lega.

(Gabriele Bartoloni, p. 3)

Tale forma si configura come calco strutturale dell'anglo-americano vaccine certificate, come si vedrà più avanti. (\$ 9.2) 


\section{Altri traducenti: passaporto vaccinale (e ingl. vaccine passport)}

L'analisi de "la Repubblica" consente di rilevare ancora un altro traducente, quale passaporto vaccinale, anch'esso ricordato da Marazzini (§§ I.2; I.2.I).

Dopo aver votato contro il parere del governo sul passaporto vaccinale, Salvini rilancia, abbraccia le posizioni care ai No Vax. (Gabriele Bartoloni, p. 3)

Ancora più numerose le attestazioni in "Google libri ricerca avanzata", tra cui:

(i) Emiliano Bezzi, Pasquale Liberatore 2021: 2.5. L'obbligo vaccinale negli Stati Uniti. 2.6. Il passaporto vaccinale negli Stati Uniti. (Digital green pass. Questioni giuridiche sottese, Key Editrice, p. 7)

(ii) Marco Annoni 202r: Questo costituisce una ragione per esentare questi gruppi dalla richiesta di passaporto vaccinale. (Etica dei vaccini: Tra libertà e responsabilità, Donzelli)

(iii) Giorgio Fiorentini 202I: si intensificherebbero (magari con un passaporto vaccinale), molte fasce deboli e fragili sarebbero vaccinate più velocemente. (Tutte le imprese devono essere sociali. Profitto \& impatto: sostenibilità per il successo, FrancoAngeli editore)

(iv) Stefano Nasetti 202r: Il pass è necessario per " muoversi liberamente" sul territorio nazionale e comunitario (poiché equivale al "passaporto vaccinale" istituito dall'Unione Europea, a sua volta richiesto per muoversi tra i Paesi del vecchio continente... (Fact Checking. La realta dei fatti, la forza delle idee, ed. Stefano Nasetti, p. I76)

(v) Marco Pizzuti 202r: impedire le discriminazioni dei non vaccinati e che non deve essere introdotto nessun passaporto vaccinale. Il Consiglio d'Europa va ricordato, è l'ente internazionale il cui scopo è promuovere la democrazia, i diritti umani. (Pandemie non autorizzate: I retroscena di un evento epocale, ed. Il Punto d'incontro)

(vii) Giusy Amato 202r: TINA: Non è obbligatorio, ma se non lo fai non ti danno questa specie di passaporto vaccinale di cui si parla. Quindi se non vuoi vivere da reclusa ti devi vaccinare e "vincere" il permesso alla libertà, altrimenti addio viaggi. (La quarantena delle donne: Monologhi brillanti, ed. Youcanprint)

(viii) Beatrice Silenzi 202r: Ora bar e ristoranti sono pieni ed è tramontata l'idea del passaporto vaccinale. Lei collabora da tempo con il dottor Fabio Franchi, uniti in questa battaglia, apertamente avversata dai media mainstream che vi definiscono - in modo dispregiativo - "Negazionisti del virus"... ( Il Pensiero degli altri. Come sono passata dal mainstream alla libera informazione, Bibliotheka)

(ix) Stefano Allievi 2021: passaporto vaccinale ne sarà uno strumento: ed è probabile che ne inventeremo altri, legati a quelle che in introduzione abbiamo definito le pandemie (o i rischi di pandemie) prossime venture. Non è difficile immaginare nuove... (Torneremo a percorrere le strade del mondo: Breve saggio sull'umanità in movimento, Utet)

(x) Andrea Crisanti, Michele Mezza 2021: Gli stessi hanno allora tentato di legare l'uso dell'app a degli incentivi, in pratica un'obbligatorietà mascherata proponendo che a Immuni sia collegato il rilascio del passaporto vaccinale. Ma siamo ancora nelle pure suggestioni. (Caccia al virus, Donzelli)

Tale forma si configura come calco strutturale dell'ingl. vaccine passport, come si vedrà più avanti § 9.I.

\section{I. Il traducente passaporto sanitario}

Un ulteriore traducente ne "la Repubblica" è passaporto sanitario:

(i) Brunetta parla del passaporto sanitario come di una misura «geniale» perché aumenta il costo «sia psichico che monetario» per "gli opportunisti contrari al vaccino". (Alberto Flores d'Arcais, p. 2)

"Google libri ricerca avanzata" rimanda peraltro a non poche attestazioni al riguardo, con diversi valori semantici, già all'inizio del 'goo, per es. 
(ii) 1906: il passaporto sanitario che affida ai municipi la sorveglianza degli individui sospetti portatori di bacilli, li proteggerà contro il colera facilitando la profilassi urbana. (Annali di medicina navale, p. II8)

E poi nel corso del 2020:

(iii) Leonardo Facco, Gruppo Lao Tsen 2020: I turisti che vogliano visitare l'Italia dovranno probabilmente munirsi di un passaporto sanitario che ne attesti la non contagiosità. Stringersi la mano o darsi un bacio non sarà più possibile. (Coronavirus: stato di paura. La storia controversa e documentata di una pandemia, Goware \& Tramedoro, p. iii)

(iv) Mikos Tarsis 2020: Aldilà degli enormi interessi economici che muovono i promotori del Great Reset, l'imposizione della vaccinazione si accompagnerà all'obbligo di un passaporto sanitario e di un ID digitale, con il conseguente tracciamento dei contatti di tutta la popolazione mondiale. (Diario di Facebook: (2017-2020), Amazon, p. 376)

\subsection{Un traducente originale: lasciapassare sanitario}

Un traducente originale all'interno della polemica contro il green pass è il lasciapassare sanitario:

"alcune delle ragioni della mia netta opposizione a un lasciapassare che non é uno strumento sanitario ma costituisce l'ennesima implementazione dell'esperimento biopolitico in corso da molto tempo nelle nostre società e che dall'epidemia Covidrg-Sarsz ha ricevuto un decisivo impulso". (A. G. Biuso)

\subsection{Un traducente "prototipico": passaporto verde}

Un ulteriore traducente, calco strutturale prototipico di green pass, è passaporto verde. Una scorsa a "Google libri ricerca avanzata" consente di attestare un uso risalente al $\mathbf{1 9 2 0}$, in ben altro contesto:

Italy. Commissariato all'emigrazione I920: "Con decreto I3 agosto I920 è stato soppresso il passaporto verde speciale per i cittadini dei paesi redenti, ai quali si rilascia il passaporto ordinario colle norme vigenti per i cittadini italiani. Il cambio sulla lira oro effettivo. (Bollettino dell'Emigrazione, vol. 19, p. 537)

Il lettore paziente troverà in Google altri ess. successivi, con riferimento a passaporti non vaccinali e vaccinali.

\subsection{Carta verde}

In limine da segnalare il composto carta verde (illegale), apparso sulla stampa e in TV, con c. 780.000 risultati in Google, calco dell'ingl. green card (I969, Merriam-Webster's Collegiate) che si aggiunge al precedente significato indicante 'il permesso di soggiorno per stranieri che vivono e lavorano in USA' (cfr. più avanti § 7).

\section{Il Green nel CoLIWeb}

Un'occhiata al corpus del CoLIWeb, curato da Marco Biffi, aggiornato al 23.I.2020 (giusto a ridosso del Covid), disponibile in rete, ricco di 33.789 lemmi ("lemma"), 763.048.374 occorrenze ("words"), I.54I.II4 forme ("word"), riguardante 2.35I.509 "documents", consente di verificare l'assenza si di Green Pass, ma ben 5.798 occorrenze di green ( 58 pp.) in vari sintagmi, tra cui green economy (605 occorrenze) 
e il su citato green card ( 38 ess.); il lemma pass appare a sua volta I.o74 volte (II pp.). Quanto ai traducenti italiani su visti,

a.) certificato digitale occorre 44 volte;

b) passaporto verde, riferito per lo più all'agricoltura, appare 7 volte;

c) certificazione verde, con riferimento all'architettura, è presente una sola volta: "acque grigie e acque piovane, che contribuisce alla certificazione verde dell' edificio e consente un risparmio di acqua potabile", gli altri 8 traducenti essendo assenti.

\section{Frequenza di Green Pass e dei traducenti/equivalenti e correttezza normativa}

Volendosi porre il problema del rapporto in termini di vitalità e diffusione dei composti su citati, Google (I 8 settembre 202I) consente di accertare la seguente classifica, in ordine decrescente:
I. Green Pass appare circa I.730.00o.ooo risultati
II. certificato Covid circa 6r.900.00o risultati
III. certificazione verde circa 43.200.00o risultati
IV. certificazione verde Covid-19 circa 38.700 .000 risultati
V. certificato digitale circa 26.800 .000 risultati
VI. certificato vaccinale circa I2.600.00o risultati
VII. certificato digitale Covid circa 9.8Io.0oo risultati
VIII. passaporto verde circa 6.580.000 risultati
IX. passaporto sanitario circa 3.360 .000
X. passaporto vaccinale circa 922.000 risultati
XI. carta verde con circa 780.000 risultati
XII. lasciapassare sanitario circa I88.0oo risultati

Una forma, la prima (Green Pass), decisamente maggioritaria rispetto alle altre, ma - normativamente tutte corrette, in quanto ricorrenti in contesti e presso parlanti colti e mediamente colti.

\section{La famiglia di green nella lessicografia}

L'entrata in italiano di Green Pass con la sua schiacciante frequenza è stata inevitabilmente agevolata dalla presenza di precedenti stranierismi con green, ben 5.798, come accennato ( $§ 5)$, le occorrenze nel citato CoLIWeb.

Per es. il Garzanti 2020 lemmatizza ben 8 lessemi: green ${ }^{\mathrm{I}}$ agg., green ${ }^{2}$ agg. e s.m. sport., green economy ("sin. economia verde"), greening, green paper, greenwashed, greenwashing, greenway.

Lo Zingarelli 202I registra 7 composti con datazioni sotto 4 lemmi: green agg. (1972) con green tax 'ecotassa', green economy 'economia verde' (1987), green s.m. sport con green keeper sport., green shoe (1994), green way (1999).

De Mauro (200o) riporta tre voci: green s.m. sport. (1983), greenkeeper sport. (I983), green shoe (I990). Il Sabatini-Coletti 2007 accoglie green agg. e s.m., green belt (I989) e greenkeeper (I989).

Il Devoto-Oli-Serianni-Trifone 2019 lemmatizza solo green agg. e s.m. (I983) e green economy (I987), a cui riserva la rubrica (neopuristica) "per dirlo in italiano", dove si osserva che "viene oggi spesso sostituito dal calco italiano economia verde", e dove si cita pure green car 'automobile verde' i.e. ecologica.

Il Merriam-Webster's Collegiate Dictionary $\left(2003{ }^{\mathrm{II}}\right)$ da parte sua lemmatizza non meno di 73 lemmi con green, tra cui il green card datato I969, cosi detto dal colore del documento, definito "an identity 
card attesting the permanent resident status of an alien in the U.S", alla base di altri analoghi composti del Green Pass, e presente nel CoLIWeb con 38 ess. (cfr. § 5).

\section{I. Produttività del greenpassare}

E chiudiamo con l'italiano in Italia sottolineando la produttivita del nostro composto nominale, che ha dato luogo a un verbo, l'ibrido greenpassare, in prima pagina nel citato "Il Fatto quotidiano":

"Altro che controlli Greenpassano tutti".

\section{Il Covid pass, certificato Covid, passaporto covid, (ecc.) nella Svizzera italiana}

Per quanto riguarda i dati sul green pass nella Svizzera italiana (Canton Ticino), disponiamo di 4 informanti.

L'informante n. I, residente in Svizzera, mi fornisce 8 designazioni, di cui 4 quelle su viste (§ 6), ovvero:

"[II] certificato covid, [VI] certificato vaccinale, passaporto covid, [X] passaporto vaccinale, ma anche covid pass $\mathrm{e}$ [I] green pass (quando si parla dell'Italia), o anche solo certificato".

Osserviamo che, riguardo a covid pass e a passaporto covid, entrambi assenti nella lista delle precedenti designazioni (§ 6), Google (2r.9.2I) indica rispettivamente "circa I.660.00o.ooo risultati" e "circa 4.390.000".

Passando all'uso personale, questo l'outing dell'informante n. I:

"Quanto a me, tendo a dire passaporto covid, o parlando semplicemente pass (hai il pass?)" come nell'uso francese (cfr. § I3.I).

Un secondo informante del Canton Ticino mi fornisce una descrizione, con ulteriori dati e con documentazione che arricchiscono la precedente, in tutto sei designazioni di cui tre su già viste (§ 6):

"qui si chiama semplicemente [II] 'certificato COVID', abbreviato in 'certificato'", specificando che " 'Certificato' è il termine usato comunemente nella conversazione (il contesto attuale rende la specificazione non necessaria)". Il tutto in conformità con le indicazioni della precedente fonte.

Un terzo informante del Canton Ticino osserva: "Confermo che nell'uso ufficiale si ricorre al termine 'certificato' o 'certificato covid".

Lo stesso informante aggiunge: "Anche nei media ticinesi, tendenzialmente si parla di 'certificato'. Si trovano però anche casi di 'pass' (con o senza 'covid') soprattutto nei titoli (allego un esempio dal giornale "La Regione" di oggi, e qui un es. dal portale Tio: https:/www.tio.ch/svizzera/attualita/I536457/certificato-universita-studenti-via-pass".

L'informante n. 2 del Canton Ticino arricchisce ancora i dati, sulla scorta di "Termdat, la banca dati della terminologia plurilingue della Confederazione", dove ha "ritrovato occorrenze in italiano di 'certificato verde digitale' o [ ] [III] 'certificazione verde" ma, se ho visto bene, sono sempre riferiti al documento europeo". "C'è un'occorrenza di 'certificato verde digitale' riferito alla Svizzera, - precisa ulteriormente la stessa fonte - ma categorizzato come 'disusato'."

Osservo che la designazione certificato verde digitale, assente nella lista del $\S 6$, è documentata in 
Google (2I.9.2I) con circa I2.00o.ooo risultati.

\section{I. Il Green pass nel Canton Ticino}

Per quanto riguarda l'anglicismo [I] green pass, l'informante n. 3 del Canton Ticino puntualizza che "'pass' o [I] 'green pass' non si trovano per es. nei comunicati stampa del Cantone Ticino".

L'informante del Canton Ticino n. 2 aggiunge ancora:

"Ti allego per finire un articolo del portale di informazione internazionale della radio e televisione di Stato, dove compare anche [I] 'green pass' tra parentesi come esplicitazione di [II] 'certificato COVID' (e l'espressione in inglese ricorre più avanti anche nel corpo dell'articolo). Questo uso è probabilmente motivato dal carattere internazionale del portale:

https://www.swissinfo.ch/ita/il-certificato-covid-pu\%C3\%B2-attendere/469I4072".

Un quarto informante del Canton Ticino allega un link con foto di un articolo del "Corriere del Ticino" dove viene riferito di un "Corteo a Bellinzona contro il COVID-Pass"; "nell'immagine si vede un manifestante con un cartellone con STOP GREEN PASS": https://www.cdt.ch/ticino/corteo-abellinzona-contro-il-covid-pass-GB465009I.

Ancora l'informante n. 3 cosi testimonia:

"Per una mini rassegna di forme nei media (ma anche nel parlato di intervistati), si può guardare l'edizione de "Il Quotidiano" di lunedi 20 settembre, dal minuto 2.46 (servizio sulla riapertura dell'anno accademico all'USI e alla SUPSI: https:/www.rsi.ch/lai/programmi/informazione/il-quotidiano/): nel parlato dei giornalisti occorrono 'certificato' o 'covid pass'; tra gli intervistati (ma vi sono anche studenti italiani) si trova anche 'green pass'. La petizione degli studenti (si vede l'immagine nel servizio) è intitolata "No all'obbligo di green pass in SUPSI".

E così ribadisce:

"Confermo anch'io di aver già sentito nel parlato (non solo giovanile) anche nella Svizzera italiana si sente anche 'green pass', con riferimento al certificato svizzero, molto probabilmente preso in prestito dall'italiano d'Italia":

"La petizione degli studenti (si vede l'immagine nel servizio) è intitolata "No all'obbligo di green pass in SUPSI".

\subsection{Le designazioni della pandemia nel Canton Ticino}

Le designazioni sulla pandemia circolanti nella Svizzera Italiana alla fine sono per frequenza le seguenti (in asterisco quelle assenti nell'uso in Italia, § 6):

I. *Covid pass (circa I.66o.ooo.ooo risultati)

I.a. Pass

II. Certificato Covid (circa 6I.9oo.ooo risultati)

II.a. Certificato

III. Certificato vaccinale (circa I2.600.00o risultati)

IV. *Passaporto covid (circa 4.390.ooo risultati)

V. Passaporto vaccinale (circa 922.000 risultati)

VI. *Certificato verde digitale ("disusato in Svizzera")

VII. Certificazione verde (ma "riferito al documento europeo") 
VIII. Green Pass (assente "nei comunicati stampa del Cantone Ticino"; prestito dall'italiano, "Stop Green Pass"; "obbligo di green pass").

\section{Il green pass anglofono}

Nel mondo anglofono il green pass non sembra godere molta fortuna, assente anche nell'Oxford English Dictionary (OED) (on line), tanto da far sospettare la presenza di uno pseudo-anglicismo.

\section{I. In Inghilterra: vaccine passports (> it. passaporto vaccinale)}

Come mi informa una nativo-anglofona, "I'm afraid here is no entry for green pass in the OED. The government are trying to get something similar (which they call vaccine passports) so as to control discotheques etc. but there is too much opposition to them". Il composto inglese si configura quindi alla base quindi della dizione passaporto vaccinale di cui sopra ( $\left.\S_{4}\right)$.

Un secondo nativo-anglofono conferma quanto sopra: "Come sospettavo "green pass" non c'è nell'OED, ma non c'è neanche il "vaccine passport", termine britannico per il documento equivalente, forse perché troppo recente".

E sottolinea l'estraneità dell'espressione per i britannici: "Ho il sospetto che per molti anglofoni non si può sentire 'green pass'".

\subsection{Anglo-amer. vaccine certificate (> it. certificato vaccinale)}

"Per gli Stati Uniti - ha ulteriormente precisato il mio primo informante anglofono - in rete ho trovato molti riferimenti a 'vaccine certificate' o anche 'white card', ma niente 'green pass'". Un composto quindi alla base del traducente certificato vaccinale, sopra indicato (§ 3.I.I).

\subsection{Il "Covid 19 Vaccination Record Card"negli USA}

Un attentissimo cultore di linguistica, residente negli USA, mi informa che "gli americani li chiamano "vaccination records/proof of vaccination" o semplicemente "CDC (vaccination) card", [CDC = Center for Disease Control], che è quella che rilasciano quando ci si vaccina", su cui si trova scritto: 'Covid 19 Vaccination Record Card'.

\subsection{I.I. L'excelsior pass neworkese}

Lo stesso informante aggiunge ancora: "A New York hanno fatto una app equivalente (più o meno) al nostro green pass e l'hanno chiamata "excelsior pass", ma questo nome lo conoscono solo a NYC. Una app standard a livello federale non esiste e troverebbe molta resistenza negli stati repubblicani".

\subsection{Il greenpass-card americano (I910)}

In americano il composto green pass-card è tuttavia documentabile, grazie a "Google libri ricerca avanzata", con riferimento ad altre situazioni:

A white card is issued to each member on admittance and represents exemplary conduct; this entitles 
the holder to free coming and going between the hours of 8 A.M. and 9 P.M. A green pass-card represents a modified privilege and is good only from 8 A.M. to 5 P.M... Annual Report of the State Board of Charities of the State, New York (State). State Board of Charities, I9ro, p. 199).

\subsection{Il Green Pass israeliano}

Il composto Green Pass pandemico appare comunque in altri contesti:

202I: EU excludes Covishield from 'Green Pass' list - Covishield has been excluded from the list of antiCOVID-rg jabs that are eligible to avail the European Union (EU)'s 'Green Pass'.

Green Pass, which would be available from July i onwards, can be used for unrestricted movement in all EU member states for business and tourism purposes. (Current Affairs June 2021, p. 42).

Il composto sembra particolarmente frequente in ambito israeliano:

Stella Immanuel 202I: 2. Miriam Fauzia, "Fact Check: Israel Launching 'Green Pass' for Citizens Vaccinated Against COVID-I9," USA Today, updated March 3, 2021". (Let America Live. Exposing the Hidden Agenda Behind the 2020 Pandemic, p. 24I).

202I: Israel launches "green pass"• Israel has launched a coronavirus "green pass" system, which allows people who have been vaccinated to access public facilities - Israelis who have got both Pfizer vaccine shots and those who have recovered from the virus get a "Green Pass" certificate in the form of a QR code or printout". (Current Affairs February 2021 E-Book!, p. 39).

Ruwantissa Abeyratne 202I: Israel introduced a digital health pass, known as the "Green Pass" this February. Vaccinated people and certain recovered COVID-I9 patients can download an app (or print a certificate with a QR code) which certifies their health status to gain entry into various facilities and venues throughout the country. (Air Transport and Pandemic Law. Legal, Regulatory, Ethical, and Economic Issues, Springer, p. 200).

\section{Io. Il Grüner Pass (ecc.) in tedesco}

Per quanto riguarda il tedesco, come mi informa un nativo-tedescofono, si riscontra il composto Grüner Pass, con altri equivalenti:

"Mentre in Italia Green Pass sembra ormai il termine ufficializzato [...], in Germania non siamo ancora a quel punto. Oltre a Grüner Pass si trovano ancora: Grüner Covid-Pass, Digitaler Impfpass, Digitaler Corona-Impfpass, Corona-Impfpass, e forse altri ancora".

Una seconda fonte nativa mi arricchisce ulteriormente l'inventario dei termini:

"mi sembra che per il momento il termine 'green pass' venga riferito solo alla situazione all'estero, mentre in Germania si parla piuttosto di 'Impfzertifikat' o 'Impfnachweis'".

\section{Io.I. Impfnachweis, Impfzertifikat (ecc.) in Austria}

Per quanto riguarda l'Austria, la situazione è analoga, ma non proprio identica rispetto alla Germania, stando alle informazioni di un terzo informante nativo.

Il calco è assente: "da noi non si parla di 'green pass', per quanto mi risulta, nemmeno in traduzione letterale. (Ma l'ho sentito prima delle vacanze quando si parlava di quell'iniziative dell'UE.)".

Quanto all'uso reale: "Generalmente si usa 'Impfzertifikat', cioè 'certificato di vaccinazione', mi sembra", come quindi in Germania.

Passando alla propria esperienza: "Il mio certificato è un foglio $\mathrm{A}_{4}$ che ogni vaccinato può stampare e 
portare con sé".

E poi ancora: "Altri usano l'"Impfpass" [i.e. 'pass(aporto) di vaccinazione'], cioè quel libriccino in cui sono annotate tutte le vaccinazioni di una persona, ma che è di colore giallo"; 'certificato internazionale di vaccinazione', stando al Nuovo diz. di tedesco di L. Giacoma-S. Kolb (Zanichelli $\left.2009^{2}\right)$.

Lo stesso informante, in una seconda e-mail, ha cosi arricchito i dati sul tedesco dell'Austria:

"Caro Claudio, ho fatto una piccola ricerca su siti austriaci di internet sulle denominazioni del green pass, e trovo le seguenti frequenze (sempre nel contesto dell'articolo determinativo):

der Impfnachweis 2.540

das Impfzertifikat 932

der Green Pass 213

der Grüne Pass I8.400".

Con l'importante osservazione che "Il predominio di grüner Pass è dovuto senz'altro al predominio in internet delle pagine ufficiali, che utilizzano spesso la parola dell'UE in traduzione tedesca".

"Nell'uso comune, si parla piuttosto - ribadisce ancora la mia fonte - di Impfnachweis o Impfzertifikat", ovvero 'attestato di vaccinazione' e 'certificato di vaccinazione', Impf(ung) 'vaccinazione'.

\section{Io.2. Il Covid-Zertificat nella Svizzera tedesca}

Riguardo al tedesco nella Svizzera tedesca, lo stesso informante della Svizzera francese sobriamente mi segnala che "prevale Covid-Zertificat".

\section{Il Certificado Covid Digital in spagnolo}

Per lo spagnolo, un informante residente in Spagna, cosi mi chiarisce:

"Come ben sai la cultura spagnola è tradizionalmente piuttosto refrattaria (anche se sempre meno) agli anglicismi e in generale ai forestierismi. Quindi qui si tende sempre a ricorrere a soluzioni magari meno sintetiche ma autoctone e trasparenti, quindi si parla di "Certificado COVID Digital" https://cvd.sanidad.gob.es/cvdcovid/cvdcovid-formulario/index.xhtml>.

Mi ha quindi allegato un modello del "Certificado COVID Digital", che ha un titolo bilingue anglospagnolo, analogo a quello italiano su indicato (§ I.I.I):

"EU Digital Covid Certificate

Certificado Covid Digital de la UE

Vaccination - Vacunación".

Nel Corpus del Español del Siglo XXI (CORPES), relativo allo spagnolo europeo e americano, il lessema Covid appare in 304 occorrenze, ma nessun es. di Green Pass.

L a "versión actual es la 0.94, publicada en el mes de julio de 202I. Cuenta con más de 327000 documentos que suman ya unos $35^{\circ}$ millones de formas ortográficas, procedentes de textos escritos y de transcripciones orales". Il lessema Green a sua volta compare invece in vari altri sintagmi tra cui green card ("39 casos en 29 documentos"). Il lemma pass è presente i69 volte; ma - stranamente nessun es. di "Certificado Covid Digital (de la UE)", né di "EU Digital Covid Certificate". 


\section{II.I. "Certificado" vs "pasaporte"}

"In realtà in un primo momento si differenziava - precisa ulteriormente la mia fonte per lo spagnolo europeo - fra "certificado" e "pasaporte", nel senso che il primo attestava che ci si era vaccinati (di fatto si generava automaticamente dopo essere stati vaccinati) mentre il secondo teneva conto (come, credo nel caso del green pass) dei tempi necessari perché il vaccino sia di fatto efficace. Poi si è solo più parlato (a quanto mi risulta) del "certificado...", che è quello che ho fatto io e che mi è servito perfettamente nelle mie vacanze in Italia quest'estate (il codice a barre viene di fatto riconosciuto in Italia come green pass)".

\section{I2. Il certificat de vacunació in catalano}

Un informante catalanista mi segnala sobriamente che il green pass "si dice certificat de vacunació". Una nativo-catalanofona mi conferma il dato, segnalandosi una situazione diglottica: parlato (varietà bassa) certificat de vacunació vs scritto (varietà alta): Certificat COVID digital de la UE:

"normalmente in catalano viene chiamato 'certificat de vacunació', anche se il nome ufficiale che compare sul sito è 'Certificat COVID digital de la UE'".

\section{Il Green Pass in francese}

Stando a Google, il Green Pass non sembra invece molto diffuso in francese. Riporto giusto appena due attestazioni:

(i) En vue de la saison touristique, l'exécutif avait plaidé pour «un green pass», une sorte de passeport sanitaire pour les touristes, idée fraîchement accueillie et abandonnée (Guide du Routard Corse 202I, Hachette, p. 402).

(ii) Food and Agriculture Organization of the United Nations, International Union for Conservation of Nature 2021: le système du «Green Pass», une certification sanitaire des produits de base qui offre la possibilité d'officialiser et de faciliter les mouvements et le commerce transnationaux du bétail dans la région (Pavanello, 2010). (Franchir les frontières. Dispositions juridiques et ... p. 59).

\section{I3.I. Il passe sanitaire / pass sanitare / pass nell'uso comune}

Per quanto riguarda l'uso comune in francese, una nativo-francofona mi informa invece che è corrente "con un triste aggettivo relazionale" l'espressione "passe sanitaire".

Sulla scorta di "Google libri ricerca avanzata", possiamo quindi documentare tale uso con varie attestazioni, tra cui:

(i) Didier Houssin 2021: pour permettre [...] la reprise d'activités aujourd'hui interdites du fait du confinement, ce «passeport ou passe sanitaire», attestant de la vaccination, voire d'un test virologique négatif ou de la présence d'anticorps contre le virus, [...]. (L'Ouragan sanitaire. Comment sortir de la pandémie du Covid-19 et préparer l'avenir, Odile Jacob)

(ii) Jean-Philippe Derenne 202r: Stratégies vaccinales. Passe sanitaire et vaccination obligatoire (Covid-19: un seul monde, Odile Jacob).

(iii) Hubert Delpech 202r: Aujourd'hui face au quatrième assaut de la pandémie, l'affirmation de la combattre TOUS ENSEMBLE semble s'éloigner de certains esprits, opposants politiques, réfractaires entêtés, ennemis du passe sanitaire obligatoire, considéré comme une atteinte aux libertés individuelles. 
(Sortir de sa caverne. En voyage et itinérance)

(iv) Dominique Auzias, Jean-Paul Labourdette 202r: Nous avons réduit le nombre de scènes à deux avec des places assises, les festivaliers étaient bien entendu masqués et ont dû présenter leur passe sanitaire. Mais le concept qui a eu le plus de succès, c'est le click and collect pour le bar et la restauration. (REIMS 2022 Petit Futé)

(v) Catherine Lison-Croze 202I: En décembre 2020, Emmanuel Macron a décidé, seul, que la vaccination ne serait pas obligatoire, puis décrété en juillet 202I, qu'elle le serait par le biais du passe sanitaire. Sous peine de mort sociale. (Cinquiéme République, la derniére vague. Projet de nouvelle constitution, Librinova).

Un secondo informante, residente in Francia, mi conferma tale dato, nella variante pass sanitare e pass con ulteriore documentazione:

In francese il termine consacrato per il green pass è 'pass sanitaire' (un'attestazione tra le tante di oggi: https://www.bfmtv.com/politique/un-projet-de-loi-pour-proroger-le-pass-sanitaire-presente-le-Izoctobre-en-conseil-des-ministres_AD-202I09200I46.html).

E ancora:

Direi che tutti (me compreso) usano 'pass sanitaire' o per antonomasia 'le pass'.

Ovvero il link illustra:

"UN PROJET DE LOI POUR PROROGER LE PASS SANITAIRE PRÉSENTÉ LE i3 OCTOBRE EN CONSEIL DES MINISTRES" di François de La Taille avec AFP, Le 20/09/202I à Io:4I", con altre cinque occorrenze del composto nel corso dell'articolo:

(i) "La prolongation de l'utilisation du pass sanitaire sera débattue à l'Assemblée avant la fin du mois d'octobre";

(ii) "Un projet de loi pour proroger le pass sanitaire au-delà du 15 novembre 'est en cours d'élaboration"';

(iii) "Vers un pass sanitaire local?";

(iv) "Le pass sanitaire, accélérateur de vaccination";

(v) "Depuis l'instauration du pass sanitaire, le gouvernement revendique les bons chiffres en matière de vaccination, avec le franchissement du seuil de 50 millions de primo-vaccinés vendredi".

E due occorrenze di pass, accanto a certificat de vaccination:

(i) "Le pass, un certificat de vaccination ou un test négatif au Covid, exigé notamment pour se rendre dans les cafés et restaurants ou prendre les transports de longue distance, suscite un mouvement de contestation dans la rue";

(ii) "Une nouvelle mobilisation a eu lieu samedi contre le pass ou la vaccination obligatoire des soignants et de certaines professions en contact avec le public."

\subsection{Il pass sanitaire (ecc.) nella Svizzera francese}

Per il francese parlato in Svizzera, un informante li residente mi segnala ben tre varianti:

I) pass sanitaire (con l'anglicismo grafico)

2) certificat covid

3) pass covid (con l'anglicismo grafico) 


\section{I4. Il passeport vaccinal / passeport sanitaire ecc. in Canada (Quebec)}

Per quel che riguarda l'uso in Canada, con riferimento al Quebec, un collega canadese mi informa: "Qui in Québec diciamo 'passeport vaccinal'". "Questa è la parola utilizzata nella stampa, in tv e anche nel linguaggio comune".

Mi indica quindi due preziosi link. Uno relativo a Usito, un dictionnaire conçu au Québec pour tous les francophones et francophiles intéressés par une description ouverte du français. Une réalisation de l'Université de Sherbrooke. Il quale sotto il lemma passeport riporta il composto passeport vaccinal definito "document officiel attestant qu'une personne est adéquatement vaccinée et lui permettant notamment de voyager ou de prendre part à des activités autrement jugées à risque de diffusion épidémique".

Il composto è distinto dal passeport sanitaire a sua volta definito "document officiel comportant des informations relatives à la vaccination (type de vaccin, nombre de doses, etc.) et au dépistage (type de test, résultats, etc.), et permettant notamment à son titulaire de voyager ou de prendre part à des activités autrement jugées à risque de diffusion épidémique".

Ma lo stesso Usito precisa anche che nella stampa i due composti sono intercambiabili: "Dans la presse, les expressions passeport sanitaire et passeport vaccinal sont souvent synonymes".

Il secondo link riguarda invece l'"Office québécois de la langue française", che riporta la voce passeport vaccinal con analoga definizione a quella di Usito, ma non contrapposto a passeport sanitaire, anzi inserito tra i "Termes privilegiés" costituiti da "passeport vaccinal, passeport sanitaire, passport immunitaire, laissez-passer sanitaire".

L'Office" da parte sua prende - puristicamente - le distanze dalle scelte del francese esagonale "en Europe francophone" (§ I3.I), indicando come "Termes déconseillés" passe sanitaire e pass sanitaire, in quanto "la résurgence de cette forme vieillie [passe] [...] principalement attribuable à l'anglais", a sua volta "l'élément pass ayant été emprunté directement à l'anglais".

L'Usito in maniera invece descrittiva aggiunge: "En France, on emploie surtout la forme critiquée pass sanitaire (n. m.) et sa francisation passe sanitaire (n. m. ou f.), qui a fait l'objet d'une recommandation officielle".

\section{I4.I. Il vaccine passport ecc. nell'inglese del Canada}

Ancora l'"Office" per l'inglese in Canada segnala sinteticamente non meno di sei termini: "vaccine passport, vaccination passport, sanitary passport"; "On trouve également dans l'usage les termes vaccine pass, vaccination pass et sanitary pass".

\section{I5. Il "passe sanitário" (ecc.) in portoghese (europeo e brasiliano)}

Riguardo al portoghese (europeo) la soluzione terminologica, stando alle informazioni della mia fonte, nativo-lusofona, è analoga a quella francese:

"Passe sanitário;

Certificado de vacinação;

Certificado de vacinação Covid; 
Certificado Covid".

In Google appare anche: "pasaporte sanitário".

Per il portoghese brasiliano invece un'altra fonte nativa mi segnala il composto trimembre (o polirematica) "certificado de vacinação".

\section{I6. Il green pass in romeno}

Stando a una mia fonte, "green pass in romeno è 'certificatul verde' (forma di citazione con l'articolo posposto $(u) l)$; anche green passul !".

\section{Il certifikatë vaksinimi, certifikatë covid in albanese}

Riguardo all'albanese tre diversi informanti, con diversa ricchezza di dati, si confermano tra di loro.

L'informante più ricco di dati premette che il green pass "in Albania non è obbligatorio, il che rende meno frequente il suo uso nel linguaggio quotidiano.".

Quanto agli ambiti d'uso, "Sulla stampa albanese si riprende alla lettera la denominazione ufficiale, quale figura nei documenti governativi e amministrativi, chiamandolo CERTIFIKATË VAKSINIMI ('certificato di vaccinazione'). In qualche occasione viene utilizzata l'espressione CERTIFIKATË COVID che però sottintende non solo l'avvenuta vaccinazione, ma anche il risultato del tampone".

Il secondo informante più sobriamente conferma quanto sopra: "la locuzione corrente è 'certifikat vaksinimi' ('certificato di vaccinazione'). Anche (ma meno) 'certifikat COVID'", e allega "una recente pagina di giornalistica on-line (con relativo link)".

Il terzo informante italiano, residente a Tirana, precisa che la dizione ufficiale in albanese è "certifikata digjitale për Covid".

\section{I7.I. Il Grinpas-i in albanese}

Quanto al green pass, il primo informante puntualizza: "Anche se il linguaggio giornalistico abbonda di anglicismi, in tal caso non ha una larga diffusione la versione trascritta del parlato - in bocca albanese!". Il calco GRINPAS-I è infatti "limitato alle escursioni di viaggiatore albanese fuori dall'Albania". Strutturalmente, spiega la mia fonte, "la 'I' finale è l'articolo determinativo post-posto maschile albanese". "L'uso di questo anglicismo, l'albanese lo adatta solitamente al suo alfabeto riprendendolo dall'inglese non dalla forma scritta, ma da quella parlata".

Il secondo informante, più attento alla circolazione interna, osserva al riguardo: "Non è usato (almeno per ora) né 'green pass' (con grafia inglese) né '* grinpas' (eventualmente, con grafia adattata)".

\section{I8. Il green pass in greco moderno}

Un informante specialista di neogreco, cosi precisa: "il Green pass in neogreco suona così e, a quanto

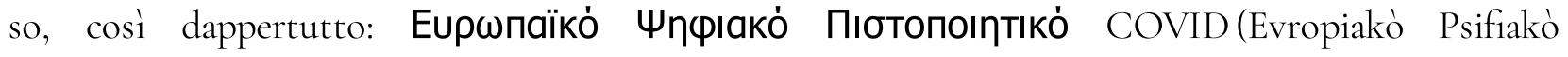
Pistopiitikò Covid) = 'Certificato Digitale Europeo Covid"'. 


\section{Il green pass in sloveno}

Quanto alla Slovenia, un collega, nativo-slovenofono, mi fornisce i seguenti dati: "uso standard: digitalno COVID potrdilo (letteralm. "attestato COVID digitale"); uso colloquiale: covidno potrdilo (letteralm. "attestato COVID").

\section{Il green pass nella ex-Jugoslavia}

Come mi informa un collega slavo, "Con la dissoluzione della Jugoslavia ci sono ora quattro stati che parlano la propria variante di quello che linguisticamente era il serbocroato, e ognuno di loro ha proclamato nella propria Costituzione di parlare lingue nazionali. Cosi ora avremmo (!) il croato, serbo, bosniaco e montenegrino".

Ovvero, "A parte questo, la università italiana considera la lingua serba e croata. In Austria: bosniaco, croato e serbo. Negli USA: bcms".

A conclusione di una sua inchiesta con amici, i dati indicatimi sono questi:

"in Croazia usiamo : Covid potvrda (attestato Covid) o Covid putovnica (passaporto Covid)";

in Serbia: Zeleni kod (codice verde);

in Bosnia ed Erzegovina: Kovid potvrda (attestato Covid);

nel Montenegro: Kovid propusnica (Lasciapassare Covid)".

Con il commento finale: "Dunque, fortunatamente senza anglicismi". Tuttavia, lo stesso informante relativo allo sloveno (§ I9), per quanto riguarda la Serbia indica due ulteriori varianti, sia l'anglicismo che il calco: in "serbo, da quanto ho potuto constatare, si usa sia Green Pass sia il suo calco serbo zelena propusnica".

\section{I. Il green pass nella LIS: una ricerca in corso}

Quanto alla LIS, ovvero alla "Lingua Italiana dei Segni", il green pass, mi dice sobriamente un bilingue segnante, "Io lo segno pass verde...". Poi in una successiva e-mail mi precisa: "non c'e ancora un segno consolidato, ma ci sono alcune proposte che devono essere ancora 'approvate' dalla comunità". Ovvero "Sono tutti segni composti che consistono in traduzioni di prestito (carta/biglietto verde; autorizzazione vaccini verde; carta governo verde)". Una ricerca in corso: "sto chiedendo in giro". Verso uno standard: "C'è la proposta di un unico segno ma la usa solo una persona".

\section{Vitalità e produttività del Green Pass}

Senza voler fare previsioni sulla durata e la fortuna del lessema Green Pass in italiano e dei suoi i2 traducenti (§ 6 certificazione verde, certificato verde, certificazione vaccinale, ecc.), si può ipotizzare che esso scomparirà quando verrà definitivamente superata la pandemia del covid, mentre attualmente la sua fortuna è anche legata alla famiglia di lessemi con green circolanti in italiano.

In conclusione, la vitalità dell'anglicismo green pass su 27 lingue e varietà qui esaminate sembra limitato ad appena due lingue, ovvero in italiano (§§ I-2) e nell'inglese israeliano (§ 9.4), nelle altre 
essendo assente: nell'italiano del Canton Ticino (§ 8), in inglese britannico (§ 9.I), in anglo-americano (§§ 9.2-9.3), nell'inglese del Canada (I4.I), nel tedesco della Germania (§ Io), dell'Austria (§ Io.I), della Svizzera (§ I0.2), in spagnolo (§ II), in catalano (§ I2), nel francese esagonale (§ I3.I), della Svizzera (§ I3.2), e del Canada (Quebec) (§ I4.I), nel portoghese europeo e brasiliano (§ I5), in romeno (§ I6), in albanese (§ I7), in neogreco (§ I8), in sloveno (§ I $)$ ), in croato, serbo, bosniaco, montenegrino (§ 20 ), nella LIS (§ 2I); in cinque essendo molto marginale (italiano del Canton Ticino §§ 8.I-8.2, albanese $\S$ I7.I, tedesco austriaco $\S$ Io.I, francese esagonale $\S$ I3, in due vigendo marginalmente il calco (tedesco austriaco § IO.I, serbo § 20).

\section{Sommario}

Riprendiamo i titoli dei §§ e sotto§§ perché il lettore possa cogliere con un colpo d'occhio il complesso percorso interlinguistico del nostro Green Pass:

\section{L'evento cruscante}

I.I. La strategia bilingue

I.I.I. Il Green Pass ufficiale

I.2. Il presidente della Crusca e il Green Pass

I.2.I. Huffingtonpost.it e "Il Resto del Carlino"

I.2.2. "Il Foglio" e la polemica delirante contro il "Green Pass"

2. Green Pass nell'uso giornalistico

2.I. Green Pass in "Google libri ricerca avanzata"

2.2. No Green Pass nell'uso giornalistico

2.2.I. Anti-Green pass

2.3. L'abbreviazione pass

2.3.I. Il No Pass

3. Il traducente certificazione verde

3.I. Il traducente certificato verde

3.I.I. Certificato vaccinale (e anglo-amer. vaccine certificate)

4. Altri traducenti: passaporto vaccinale (e ingl. vaccine passport)

4.I. Il traducente passaporto sanitario

4.2. Un traducente originale: lasciapassare sanitario

4.3. Un traducente "prototipico": passaporto verde

4.4. Carta verde

5. Il Green nel CoLIWeb

6. Frequenza di Green Pass e dei traducenti/equivalenti e correttezza normativa

7. La famiglia di green nella lessicografia

7.I. Produttività del greenpassare

8. Il Covid pass, certificato Covid, passaporto covid, (ecc.) nella Svizzera italiana

8.I. Il Green pass nel Canton Ticino

8.2. Le designazioni della pandemia nel Canton Ticino

9. Il green pass anglofono

9.I. In Inghilterra: vaccine passports (> it. passaporto vaccinale)

9.2. Anglo-amer. vaccine certificate (> it. certificato vaccinale)

9.2.I. Il "Covid 19 Vaccination Record Card" negli USA

9.2.I.I. L'excelsior pass neworkese

9.3. Il greenpass-card americano (I9I0)

9.4. Il Green Pass israeliano 
Io. Il Grüner Pass (ecc.) in tedesco

Io.I. Impfnachweis, Impfzertifikat (ecc.) in Austria

Io.2. Il Covid-Zertificat nella Svizzera tedesca

II. Il Certificado Covid Digital in spagnolo

II.I. "Certificado" vs "pasaporte"

I2. Il certificat de vacunació in catalano

I3. Il Green Pass in francese

I3.I. Il passe sanitaire / pass sanitare / pass nell'uso comune

I3.2. Il pass sanitaire (ecc.) nella Svizzera francese

I4. Il passeport vaccinal / passeport sanitaire ecc. in Canada (Quebec)

I4.I. Il vaccine passport ecc. nell'inglese del Canada

I5. Il "passe sanitário" (ecc.) in portoghese (europeo e brasiliano)

I6. Il green pass in romeno

I7. Il certifikatë vaksinimi, certifikatë covid in albanese

I7.I. Il Grinpas-i in albanese

I8. Il green pass in greco moderno

I9. Il green pass in sloveno

20. Il green pass nella ex-Jugoslavia

2I. Il green pass nella LIS

22. Vitalità e produttività del Green Pass

\section{Cita come:}

Salvatore Claudio Sgroi, //Green Pass all'Accademia della Crusca (e altrove), ovvero per una storia de/Green Pass, "Italiano digitale", XVIII, 2021/3 (luglio-settembre)

DOI: 10.35948/2532-9006/2021.11605

Copyright 2021 Accademia della Crusca

Pubblicato con licenza creative commons CC BY-NC-ND 